\title{
Onkologie-Mitteilungen
}

Bitte senden Sie alle Mitteilungen, die unter dieser Rubrik publiziert werden sollen, an Herrn Prof. Dr. med. G. Nagel, Med. Universitätsklinik, Abteilang Hämatologie/Onkologie, RobertKoch-Straße 40, D-3400 Göttingen

Onkologie Mitteilungen

- ein neues Ange-bot der Zeitschrift Onkologie

Die Zeitschrift Onkologie soil vom Oktober 1979 an durch eine neue, regelmäßig in jedem Heft erscheinende Spalte, durch die ONKOLOGIE MITTEILUNGEN, bereichert werden. Dabei handelt es sich um folgendes:

Die ONKOLOGIE orientierte ihre Leser bisher fast ausschließlich über Belange der patientenorientierten onkologischen Torschung und Praxis durch die Wiedergabe von Originalmitteilungen.

In letzter Zeit häufen sich aber nun Leserstimmen mit der Bitte um ein erweitertes Informationsangebot.

Die Krebsmedizin unterliegt zur Zeit mehr denn je einem raschen Wandel. Neue Therapien werden entwickelt, bisher unbekannte Neben-wirkungen beschrieben, das heute noch gültige Wissen wird morgen in Fortbildungskursen überholt, die Frage "Bin ich auf dem Tumor-gebiet noch aktuell ausgebildet und orientiert?” stellt sich immer mehr Ärzten in Klinik und Praxis. Optimale Tumortherapie kann nur noch treiben, wer sich permanent über die Neuerungen im Fach Onkologie auf dem Laufenden halt. Diese Information ist jedoch nur bedingt aus Originalarbeiten erhältlich - Arbeiten, die zum Teil beim Erscheinen schon wieder an Aktualität verloren haben.

Woher ist aber das Wissen über neue, ungewohnte Medikamente, über Fortbildungsveranstaltungen, über Zwischenresultate von Studien, über die ¥ertigkeit neuer diagnostischer Verfahren etc. zu bekom-men? Bisher nur mühsam aus zahlreichen Quellen, die wie z.B. Frühergebnisse von laufenden Chemotherapiestudien - auch dann oft nur sehr schwer zugänglich sind.

In den ONKOLOGIE MITTEILUNGEN werden Sie, verehrte Leser, in Zu-kunft über Aktuelles aus der Onkologie orientiert. Die ONKOLOGIE MITTEILUNGEN sind der von İhnen gewünschte Onkologie Newsletter.

\section{ONKOLOGIE MITTEILUNGEN}

\section{Editorial} worüber wird berichtet?

- $\quad$ namhafte Fachleute werden gebeten, zu einem aktuellen und kontroversen Thema Stellung zu nehmen.

Onkologie-Mitteilungen

219

Medikamente, Therapien

Neue Medikamente, Antidots und Therapieprinzipien werden vorgestellt.

Therapienebenwirkungen und deren Prophylaxe und Bekämpfung "werden bekanntgemacht . 
Alternativtherapien werden ge"werte.t.

Eta"bliertes wird gegen das Experimentelle gestellt.

Klinische Studien

In diesem Abschnitt sollen nicht nur neue Studienprotokolle, sondern auch Zwischenergebnisse laufender oder Endresultate abgeschlossener klinischer Studien im Vorabdruck publiziert werden.

- $\quad$ Studiengruppen haben Gelegenheit, über ihre Tätigkeit zu berichten. Auf Anfrage aus dem Leserkreis wird bekanntgegeben, wer wo welche Studie durchführt.

Wer Interesse an einem seltenen Tumor oder an einer sehr gezielten Fragestellung hat, kann sich in dieser Rubrik an die Kollegen in Forschung, Klinik und Praxis mit der Bitte um Meldung von Patienten, Beratung oder Mithilfe wenden.

Kongresse, Fortbildungsveranstaltungen

Jede Nummer der ONKOLOGIE MITTEILUNGEN enthält einen Kon-gress-, Fortbildungs-, Workshop- und Seminarkalender relevan-ter Veranstaltungen zum Thema Onkologie im In- und Ausland.

- $\quad$ Auf die oft schwierig zu bekommenden Anmeldetermine zu solchen Veranstaltungen und Abstract Deadlines wird hingewiesen.

Kongreßprogramme gehören zu dieser Spalte - wie auch Kurz-referate über Kongresse.

Mitteilungen der Fachgesellschaften und Studiengruppen

Fachgesellschaften und Studiengruppen, die sich schwerpunkt-mäßig onkologischen Fragen widmen, wird Raum für eigene Mitteilungen eingeräumt. Hier kann über eigene

Veranstaltungen, Berichte an die Mitglieder, Sitzungsprotokolle etc. von den Fachgesellschaften selbst berichtet werden.

Auch Laienorganisationen und Selbsthilfegruppen sollen hier vertreten sein.

Adressen, Kontaktpersonen

Es mag wünschenswert sein, einmal die Liste aller onkologischen Förderungsorganisationen oder Studiengruppen oder Re-ferenzlaboratorien zur Hormonrezeptorbestimmung im deutschsprachigen Raum usw. zu veröffentlichen. Die ONKOLOGIE MITTEILUNGEN folgen hier dem ¥unsch ihrer Leser und vermitteln Informationen.

220

Onkologie-Mitteilungen

Stellenmarkt, Stipendien

Unter dieser Rubrik finden Sie in Zukunft : Hämatologische und onkologiscbe Stellenange"bote und -gesuche. Offerten von Aus“bildungs- und ¥eit er”bildungsplät zen , Aus schr ei”bungen von Stipendien.

0NK0L0GIE MITTEILUNGEN

- ïer berichtet?

Verlag und Herausgeber der Zeitschrift 0NK0L0GIE werden sich zunäcl·ist einmal selbst urn aktuelle, allgemein interessierende Inf ormat ionen "bemühen.

- Weiterhin wird von der 0NK0L0GIE ein verantwortlich-er Herausgeber der 0NK0L0GIE MITTEILUNGEN gewählt, ¥elcLer für die 0NK0L0GIE MITTEILUNGEN relevante Informationen sammelt und 
redigiert.

Die Fachgesellschaften möchten wir noch einmal auffordern, ihre Mitteilungsspalte nach eigenem Ermessen zu nutzen und auch

- an unsere Leserschaft treten wir mit der Bitte heran, für die 0NK0L0GIE MITTEILUNGEN geeignete Beiträge einzusenden. 0NK0LOGIE MITTEILUNGEN

- aktuell

Die 0NK0L0GIE MITTEILUNGEN können ihren Zweck - nämlich aktuelle onkologische Informationen von und für onkologisch Interessierte rasch auszutauschen - nur erfüllen, wenn der Redaktionsschluß für die Mitteilungen möglichst nahe beim Erscheinungsdatum der 0NK0L0GIE liegt.

Andererseits muß eine ausreichende Zeit für Redaktion, Druck und Herstellung zur Verfügung stehen.

Dem Bedurfnis beider Seiten entgegenkommend wurde deswegen als Einsendeschluß für Mitteilungen jeweils der Termin a $\mathrm{c} \mathrm{h} \mathrm{t}$ Wochen vor Erscheinen der nächsten 0NK0L0GIE Nummer gewählt. Die Erscheinungsdaten sind jeweils:

Heft 1

20. Februar

Heft 2

20. April

Heft 3

20. Juni

Heft k

20. August

Heft 5

20. Oktober

Heft 6

20. Dezember

Verehrte ONKOLOGIE-Leser, wir hoffen, mit den 0NK0L0GIE MITTEILUNGEN das Unsere zur Verbesserung der Krebsmedizin beizutragen und freuen uns auf Ihre weiteren Anregungen und Beiträge .

München, Oktober 1979 Herausgeber und Verlag 